\title{
P02-21
}

\section{DEPRESSION AND CYTOKINES: A PSYCHONEUROIMMUNE PERSPECTIVE}

\section{L.C. Castro}

Psychiatry, Hospital de Magalhães Lemos, Porto, Portugal

Background: Cytokine-induced sickness behaviour is an adaptative response to infection. Activation of the immune system, particularly the increased activity of several cytokines, has been implicated in the provocation or exacerbation of mood disorders.

Aim: To discuss the relevance of psychoneuroimmunologic research in the understanding of the patophysiology and therapeutic targets of certain mental health disorders.

Methods: Review of the literature. MEDLINE and PubMed databases searches for peer-reviewed studies, published between 2000 and 2008, using combinations of the Medline Subject Heading terms cytokines, sickness behaviour, immune system and depression, depressive illness.

Results: Several lines of evidence support the relationship between cytokines and mood disorders. Cytokine elevations are more marked in severe depression and their activity may also relate with illness chronicity, neurovegetative features and high stress perception. It is argued that the activation of the inflammatory system, eliciting cytokine release and synthesis, provoke neuroendocrine and neurotransmitter changes, contributing to the development of depression. Some studies support that the brain cytokine system can become sensitized in response to non-immune stressors or to immune stressors occurring early in life.

Discussion: The cytokines actions in the brain can help to clarify the patophysiology of certain psychiatric disorders, including depression. The research findings show an association between the activation of the brain cytokine system and depression, although the precise relationship between sickness behaviour and depression is still not clear. Further research in such a promising area of study is needed and can contribute to new therapeutical targets and a broader understanding of psychiatric disorders. 